\title{
Réforme curriculaire et construction de savoirs issus de psychologie. Ressources et obstacles dans la chaîne transpositive
}

Curricular reform and psychological knowledge. Resources and obstacles in didactics transposition

Nathalie Panissal et Emmanuelle Brossais

\section{OpenEdition Journals}

Édition électronique

URL : http://journals.openedition.org/educationdidactique/1300

DOI : $10.4000 /$ educationdidactique. 1300

ISSN : 2111-4838

Éditeur

Presses universitaires de Rennes

\section{Édition imprimée}

Date de publication : 31 mai 2012

Pagination : 69-84

ISBN : $978-2-7535-1872-8$

ISSN : 1956-3485

\section{Référence électronique}

Nathalie Panissal et Emmanuelle Brossais, « Réforme curriculaire et construction de savoirs issus de psychologie. Ressources et obstacles dans la chaîne transpositive », Éducation et didactique [En ligne], 6-1 | 2012, mis en ligne le 02 mai 2014, consulté le 08 décembre 2020. URL : http:// journals.openedition.org/educationdidactique/1300; DOI : https://doi.org/10.4000/ educationdidactique.1300 


\title{
RÉFORME CURRICULAIRE ET CONSTRUCTION DE SAVOIRS ISSUS DE PSYCHOLOGIE
}

\author{
RESSOURCES ET OBSTACLES DANS LA CHAÎNE TRANSPOSITIVE
}

\author{
Nathalie Panissal (UMR EFTS, IUFM Midi Pyrénées-Uuniversité de Toulouse 2) \\ Emmanuelle Brossais (UMR EFTS, IUFM Midi Pyrénées-Uuniversité de Toulouse 2)
}

\begin{abstract}
Résumé: Cette étude transpositive traite la question des relais entre un nouveau programme de la filière technologique tertiaire (Sciences et Technologies de la Gestion) et sa mise en œuvre par deux enseignantes via le même manuel scolaire. Lapplication de cette réforme concerne de nouveaux savoirs de psychologie et une nouvelle démarche d'enseignement: Observation-Analyse-Conceptualisation (OAC). Nous étudions quatre étapes de la chaîne transpositive pour les concepts de psychologie «Attitude et comportement » qui sont l'objet de cette réforme: analyse a priori des savoirs de psychologie, analyse du programme, analyse d'un manuel et analyse de pratiques de classes de deux professeures d'économie-gestion. Lanalyse a priori fait apparaître deux types d'obstacles à la construction des savoirs issus de la psychologie. Lanalyse du contrat didactique permet d'examiner les rôles des enseignantes et des élèves dans la construction du savoir en lien avec la démarche OAC. Nous examinons la façon dont les enseignantes, les auteurs du programme et ceux du manuel se confrontent aux obstacles épistémologiques inhérents aux concepts psychologiques en question. Nous discutons la façon dont enseignantes et auteurs du manuel composent avec l'injonction de la démarche OAC, elle-même source d'obstacles.
\end{abstract}

Mots-clés : manuel scolaire, pratiques enseignantes, transposition didactique, contrat didactique, réforme STG.

Nathalie Panissal \& Emmanuelle Brossais

\section{Introduction}

L'application d'une réforme pose la question des relais entre les décisions prises par les rédacteurs du nouveau programme et sa mise en ouvre dans les classes (Brossais, Panissal, Matheron, LudwigLegardez, Legardez, \& Terrisse, 2007). En 2005, les baccalauréats technologiques tertiaires ont été rénovés dans la perspective de mieux préparer les élèves à des études supérieures de gestion en introduisant des savoirs issus des sciences humaines et sociales.

Le professeur d'économie-gestion enseignant en Sciences et Technologies de la Gestion (STG) conçoit et met en œuvre son enseignement dans sa classe en composant avec plusieurs ressources documentaires dont le manuel, qui joue un rôle important en étant un des supports de l'application d'une réforme.

Les pratiques enseignantes concordent-elles avec les ambitions du programme par le biais de l'exploitation d'un manuel dans la classe? Le manuel utilisé est-il adapté aux décisions de la noosphère? De quelle manière les savoirs issus de la psychologie sont traités aux cours des différentes étapes de la chaîne transpositive?

\section{Contexte de la réforme}

L'objectif assigné à la rénovation de la série STG vise à la démarquer plus nettement de la voie professionnelle et l'exercice immédiat d'un métier, pour la rapprocher des séries générales, en vue d'une poursuite d'études supérieures.

Un des enjeux de la réforme consiste à remplacer le terme de compétences (dans le sens où les élèves ne seront plus formés et évalués à la maîtrise de compétences) par le terme de concepts scientifiques (connaissances et contenus) et conceptualisation. La revalorisation de la filière visée par cette réforme passe notamment, par l'adoption d'une forme d'enseignement d'inspiration constructiviste, non exclusive, du nom de « démarche OAC » (Observation-AnalyseConceptualisation). Cette démarche se positionne ainsi comme un compromis entre les injonctions d'inductivisme caractérisant la série STT et les démarches plus déductives des séries générales (Legardez, 2005). Pour ses « rénovateurs » l'enseignement nouveau que l'on y dispensera se doit de permettre le développement d'instruments cognitifs généraux d'observation, d'analyse et de conceptualisation conformes à ce qu'ils se représentent être des outils à l'acquisition indispensable aux études supérieures. 
Pour l'enseignement « d'information et communication », les programmes des classes de $1^{\text {re }}$ STG spécialité "communication " introduisent de nouveaux savoirs notamment issus de psychologie. Les instructions officielles précisent qu'il convient de transmettre ces savoirs qui s'appuient sur des références scientifiquement validées et de transmettre des notions simples rigoureuses et attractives mais sans les théories qui les fondent. Il s'agit d'utiliser les concepts de psychologie pour comprendre les situations de communication. Ainsi, les enseignants de la filière, titulaires d'un CAPET ou d'une agrégation d'économie-gestion, sont-ils amenés à enseigner des savoirs entièrement nouveaux pour eux.

\section{Analyse a priori des savoirs en jeu}

Nous examinons dans cet article les concepts d'attitude et comportement. Le recours à l'enseignement de ces concepts pose d'emblée une difficulté terminologique propre à gêner la compréhension des concepts eux-mêmes.

\section{Sens commun}

Le premier obstacle vient du sens usuel d'attitude qui désigne un comportement. Par exemple, un enseignant demande à un élève perturbateur de changer d'attitude sous peine de sanction. En psychologie sociale, le terme adapté à cette situation est le comportement alors que dans l'usage commun l'attitude est souvent assimilée à une position du corps, à une manière de se tenir, voire au comportement que l'on adopte.

Il s'agit donc de remplacer chez les élèves (et leurs professeurs) des connaissances élaborées dans la vie quotidienne par des concepts scientifiques.

\section{Polysémie et pluriparadigmatisme}

La notion d'attitude reçoit des définitions nombreuses et différentes en psychologie sociale. Ainsi pour Thomas et Znaniecki (1919), l'attitude est un « état d'esprit de l'individu envers une valeur». On note la centration sur le caractère inobservable de l'attitude: « état d'esprit ». La référence à la notion de valeur met l'accent sur les dimensions normatives et socio-culturelles en lien avec les groupes où se construisent ces valeurs.

Park et Burgess (1921) et Allport (1935) visent surtout à la distinguer de la notion d'instinct en faisant valoir l'importance de l'expérience passée dans la formation des attitudes. La construction de l'attitude en relation avec des facteurs historico-culturels et psychologiques est différente d'un comportement instinctif, inné, héréditaire, accompli sans apprentissage préalable.

Pour Oskamp (1977), c'est une « disposition à réagir de façon favorable ou défavorable à un objet particulier ou classe d'objets ». On retrouve l'idée que l'attitude peut s'appliquer à des objets très divers (consommation, sexualité, OGM...). Les attitudes sont des prédispositions à répondre à des classes de stimuli par des classes de réponses; les attitudes représentent trois catégories de réponse: cognitive, affective et comportementale (Rosenberg et Hovland, 1960). La définition d'Oskamp fait apparaître la dimension unidimensionnelle de l'attitude tandis qu'elle est tridimensionnelle pour Rosenberg et Hovland, pour qui à la dimension affective s'ajoutent les dimensions cognitives (connaissances et croyances au sujet d'un objet d'attitude) et conatives (les intentions comportementales).

L'une des particularités des objets de savoir constitutifs des sciences humaines est leur plurivocité. Les définir, et surtout les appréhender correctement, présupposent de pouvoir les référer aux divers paradigmes qui ont historiquement vu le jour en psychologie; ce qui interdit de les penser en terme d'univocité de sens.

\section{Relativité du lien entre attitude et comportement}

Outre la polysémie du terme, la relation attitudecomportement fait l'objet d'importants débats en psychologie sociale. Ainsi, il existe trois " générations de travaux » qui abordent, la question du lien entre attitude et comportement: ceux qui concluent à une relation très faible, ceux qui concluent à une relation modérée, ceux qui examinent les caractéristiques des attitudes et la manière dont elles peuvent engendrer des comportements (Michelik, 2008). 
Parmi les auteurs pour qui attitude et comportement sont intimement liés: Allport (o.c.) définit l'attitude comme: "état mental et neurophysiologique, constitué par l'expérience qui exerce une influence dynamique sur l'individu, le préparant à réagir d'une manière particulière à un certain nombre d'objets et de situations » (o.c., p. 799). Les attitudes sont alors considérées comme indispensables pour expliquer les comportements. Stoetzel (1963) la définit comme une variable inférée, non directement observable: à la source des comportements individuels, l'observateur aperçoit une représentation du monde, des valeurs, qui disposent chacun à réagir aux événements d'une manière déterminée, et constituent des attitudes. Non nécessairement conscientes, celles-ci s'expriment sous forme verbale et non verbale dans les comportements. Wiggins \& Breckler (1992) définissent les attitudes comme des représentations mentales et neuronales, organisées par l'expérience, exerçant une influence dynamique sur le comportement. Pour ces auteurs, l'attitude est considérée comme une variable intermédiaire entre les facteurs psychologiques et socio-culturels qui ont conduit à la formation de cette attitude et l'action à l'égard d'un objet donné en fonction de cette attitude.

Pour ces auteurs, l'attitude permet de prédire les comportements réels et potentiels d'un individu devant une stimulation sociale, elle est un concept indispensable dans l'explication du comportement, défini comme ensemble de réactions objectivement observables.

Cependant, la prédictivité d'un comportement en lien avec une attitude est sous-tendue par la force de l'attitude. Plus une attitude est forte (accessible, importante...) plus l'individu sera amené à produire un comportement consubstantiel avec cette attitude (Michelik, 2008). De plus, il arrive que le comportement ne semble pas congruent avec l'attitude. Ainsi, une personne peut considérer que l'alcoolisme est dangereux pour la santé tout en poursuivant sa consommation d'alcool, estimant que sa consommation d'alcool ne relève pas de l'alcoolisme. Cette évaluation devient un obstacle au changement de comportement (arrêt de la consommation). L'attitude est ici composée de plusieurs éléments qui peuvent entrer en conflit: la perception des dangers de l'alcoolisme et une évaluation de sa consommation personnelle d'alcool comme non problématique. Il est donc quasiment impossible de déterminer un comportement à partir d'une seule attitude compte tenu de la multifactorialité de l'attitude.

\section{Problématique et questions de recherche}

L'application de la réforme STG concerne à la fois de nouveaux savoirs et une nouvelle démarche d'enseignement. Nous cherchons à caractériser les transformations subies par des savoirs issus de la psychologie quand ils deviennent objets d'enseignement. Cette reconstruction tient compte des demandes du public scolaire et des exigences de l'institution. Ainsi la transposition didactique désigne « le passage du savoir savant au savoir enseigné. C'est à la confrontation de ces deux termes, à la distance qui les sépare qu'on peut le mieux saisir la spécificité du traitement didactique » (Chevallard, 1985). Le processus de transposition didactique implique diverses institutions en interaction les unes avec les autres. La noosphère produit un programme (les savoirs scolaires institutionnels). Une autre institution est constituée des éditeurs et rédacteurs de manuels scolaires qui sont alors des « savoirs intermédiaires » (Legardez, 2004). Les manuels sont une première textualisation des programmes (Tavignot, 1995). La dernière étape de la chaîne transpositive est le fait des enseignants qui, partant des savoirs à enseigner, construisent leurs enseignements à « bonnes distances » des autres savoirs en mettant en œuvre leur interprétation dans la façon de faire. Ainsi, la transposition didactique externe concerne les transformations entre le programme et le manuel tandis que la transposition didactique interne (Chevallard, 1985) désigne l'adaptation que l'enseignant fait du programme et du manuel dans sa classe.

L'enseignant prend des décisions parmi un ensemble de possibles qu'autorisent la situation et ses contraintes à la fois dans la préparation des cours et dans la conduite du contrat didactique (Brousseau, 1986, Sensevy, 2001).

Dans toute situation didactique, le maître tente de faire savoir à l'élève ce qu'il veut qu'il fasse mais ne peut pas le dire pour éviter que l'élève n'ait qu'à exécuter une série d'ordres. Ce contrat fonctionne comme un système d'obligations réciproques, 
explicites et surtout implicites, qui détermine ce que chaque partenaire, enseignant et enseigné, a la responsabilité de gérer, et dont il sera d'une manière ou d'une autre, responsable devant l'autre (Brousseau, 1986). Cette notion de contrat est centrale pour aborder d'une part la façon particulière dont chaque élève interprète la situation d'apprentissage, et réagit en fonction des intentions supposées et/ou réelles de l'enseignant; et d'autre part pour définir les significations des contenus qui déterminent, même à son insu, les stratégies d'intervention de l'enseignant (Tavignot, 1995).

La réforme accorde une place centrale à l'élève en tant qu'acteur dans la construction des savoirs en particulier avec la méthode OAC. Nous nous centrons sur la notion de topogenèse qui permet de rendre compte des rôles de l'enseignant et des élèves relativement au savoir, soit les places assignées à chacun des protagonistes de la relation didactique par rapport aux tâches didactiques afin de comparer l'analyse des pratiques de classes à l'analyse d'un manuel scolaire.

L'analyse de la topogenèse rend compte de l'offre de dévolution du manuel - nous parlons de topogenèse a priori (Panissal, Brossais, Legardez, \& Ludwig-Legardez, 2007) - et de l'enseignant quand la construction de la situation par l'enseignant permet à l'élève de s'engager dans la responsabilité d'acquérir le savoir visé (Brousseau, 1986).

Nous nous demandons de quelle(s) façon(s) les auteurs du programme, les enseignants et les auteurs d'un manuel se confrontent aux obstacles épistémologiques inhérents aux concepts et de quelle façon les enseignants et les auteurs du manuel composent avec l'injonction de la démarche OAC.

\section{Méthodologie}

Nous nous centrons sur les ressources documentaires et les pratiques d'enseignement pour analyser d'une part, la transposition des savoirs « attitude » et « relation entre attitude et comportement » et d'autre part, le mode de construction des savoirs prévu par la méthode OAC.
Nous analysons deux séances de classes menées sur ces concepts en lycée technologique. Nous avons choisi deux enseignantes volontaires parce qu'elles sont expérimentées (elles enseignent dans la filière depuis plus de quinze ans) et font usage du même manuel dans une classe de première STG Information et Communication (élèves de 16-17 ans).

Les séances ont été enregistrées en octobrenovembre 2005, première année d'application du nouveau programme, et intégralement retranscrites.

Les ressources documentaires examinées sont: le programme de première STG spécialité Information-Communication et un manuel scolaire.

Nos deux indicateurs pour l'analyse transpositive des savoirs sont:

- la définition de l'attitude dans la perspective de la synonymie usuelle avec comportement et de la polysémie du terme dans un cadre pluriparadigmatique,

- la relation de prédictivité entre attitude et comportement et la multifactorialité de cette influence.

Notre indicateur pour l'analyse du contrat didactique est la topogenèse soit la place de chaque acteur du système didactique (élève et enseignant) dans la construction du savoir à apprendre. La topogenèse a priori dans le manuel et la topogenèse en situation interactive, sous l'angle de la dévolution et l'institutionnalisation, permettent de rendre compte de la mise en œuvre de la démarche pédagogique Observation-Analyse-Conceptualisation (OAC).

\section{Construction des savoirs et traitement des obstacles dans la chaine transpositive}

\section{Les choix pour la construction des savoirs dans le programme et les obstacles associés}

Les savoirs

Le programme se présente sous la forme de trois colonnes. 


\begin{tabular}{|c|c|c|}
\hline \multicolumn{1}{|c|}{ Thèmes } & \multicolumn{1}{|c|}{ SENS ET PORTÉE DE L'ÉTUDE } & NOTIONS ET CONTENUS À CONSTRUIRE \\
\hline $\begin{array}{l}\text { 2.2. Attitude et } \\
\text { comportement }\end{array}$ & $\begin{array}{l}\text { L'attitude, résultante de facteurs socioculturels } \\
\text { et psychologiques, constitue une prédisposition } \\
\text { à porter un jugement ou à adopter un comporte- } \\
\text { ment particulier à l'égard d'un objet déterminé. } \\
\text { Elle peut être prédictive de comportements, en } \\
\text { particulier professionnels ou de consommation. }\end{array}$ & $\begin{array}{l}\text { Attititude. Composantes de l'attitude: affective, } \\
\text { de lien attitude-comportement. }\end{array}$ \\
\hline
\end{tabular}

La lecture du programme place le lecteur face à la difficulté de définir la notion d'attitude sans la confondre avec celle de comportement (la notion de comportement étant définie lors du premier chapitre du programme), puis de lier les deux concepts en montrant que l'attitude peut influer sur un comportement. Jugement et comportement sont associés, les deux étant déterminés par une attitude.

La définition proposée est proche de celle d'Oskamp (1977) qui évoque «une disposition à réagir » et Allport (1935) proposant « état [...] préparant l'individu à réagir ». Tandis que pour Oskamp, l'attitude est unidimensionnelle, le programme retient l'aspect tridimensionnel de l'attitude (affectif, cognitif et conatif) proposé par Rosenberg et Hovland (1960). Ce choix témoigne d'une superposition des paradigmes.

La troisième difficulté tient à la prédictibilité entre une attitude et un comportement. La modalisation « elle peut être prédictive de comportements » rend compte du caractère non automatique de la liaison mais l'expression «lien attitude-comportement " au singulier laisse entendre une relation linéaire entre une attitude et le comportement qui lui serait associé.

\section{La démarche "OAC », une stratégie pédagogique institutionnelle}

La revalorisation passe par la préconisation d'une démarche d'enseignement dite ObservationAnalyse-Conceptualisation. Cette progression $\mathrm{OAC}$ est proche des formes de pédagogies dites «actives » traditionnellement privilégiées par les séries technologiques, soit des situations concrètes où l'élève élabore des habiletés utiles à l'action au travail. Ces pédagogies inductives partent de l'observation de cas concrets pour en inférer la règle. Elles sont valorisées dans les enseignements technologique et professionnel, tandis que les pédagogies déductives caractériseraient, de manière alternative, l'enseignement général.

Les concepts sont peu à peu construits en phase d'observation et d'analyse, ensuite ils sont intégrés en des systèmes puissants qui sont alors utilisés de manière efficiente dans toutes les situations de la vie. Or, la conceptualisation est un processus cognitiviste et constructiviste de haut niveau. Il consiste à catégoriser des objets, opération pour laquelle l'élève doit construire les propriétés essentielles du concept en question (ses attributs), pour le spécifier et établir les relations entre elles (Barth, 1993). Le programme ne donne toutefois pas d'indications explicites quant aux stratégies d'enseignement à développer pour amener les élèves à la conceptualisation, ni même une définition claire de ce que l'on entend par conceptualisation. Chaque partie préconise la démarche: Observation-Analyse-Conceptualisation et l'illustre par quelques exemples indicatifs: "Toute séquence pédagogique doit permettre aux élèves, par une synthèse adaptée, de dégager l'essentiel des notions abordées. C'est notamment par ce travail de conceptualisation que l'enseignement de communication et information prend tout son sens " (Programme, p. 10). La conceptualisation s'assimile ainsi à une synthèse à réaliser en fin de cours. Mais alors qu'est ce qui différencie la filière STG et la filière STT où il s'agissait déjà d'aborder les notions par une démarche inductive, à conclure par une synthèse en fin de séance? Les instructions officielles (2005) mettent plus l'accent sur leur volonté de se démarquer de la voie professionnelle que sur la façon d'amener les élèves vers ce processus mental de haut niveau. 


\section{Un manuel original globalement concordant avec la rénovation : écueils évités et glissement sémantique}

\section{Méthode pédagogique OAC et topogenèse a priori}

Le manuel analysé propose une formalisation de cette démarche en associant aux questions les lettres O, A ou C (Guittard et al., 2005a, p. 51). Ainsi, on note dans le premier exercice (cf. ci-après description de l'exercice): O: Quel comportement cela aurait-il tendance à provoquer chez vous?; A: Efforcez-vous de tirer les conclusions de cet exercice et $C$ : En vous fondant sur ces exemples, donnez une définition de l'attitude.

Les notions sont à élaborer en classe dans l'interaction enseignante-élèves suivant la méthode OAC, le manuel n'offre pas de définition d'attitude. Ce type d'organisation du manuel est une nouveauté par rapport aux habitudes de la filière STT, jusqu'à présent les manuels des élèves présentaient un cours structuré sur les notions étudiées. Un grand degré de liberté est laissé à l'enseignant et à l'élève pour construire les notions de ce chapitre. Cette offre de dévolution du manuel, que nous proposons de nommer topogenèse a priori, concerne deux notions du programme (attitude; lien attitude-comportement) et une notion hors programme (différence attitude et comportement).

Les savoirs en jeu

Définition attitude: polysémie, synonymie et sens commun

Un exercice ouvrant le chapitre introduit la différence entre attitude et comportement. Si elle n'apparaît pas dans les textes officiels, elle permet d'aborder la relation qui unit les deux notions d'abord en les distinguant puis en les liant. Cette distinction permet de lever l'ambiguité courante consistant à considérer attitude et comportement comme synonymes.

Mais, la notion d'attitude est assimilée à celle de jugement «quel jugement portez-vous spontanément face à ces photos? Autrement dit, quelle attitude avez-vous face à ces photos?». Les auteurs du programme reprennent - sans les nommer - Allport et Oskamp qui affirment que l'attitude influence le jugement ou le comportement. Mais les auteurs du manuel considèrent attitude et jugement comme des synonymes. Ce glissement sémantique et conceptuel peut conduire l'enseignant dans la situation de classe à exclure les comportements de type verbal ou non verbal comme l'expression d'un jugement de valeur et donc à se cantonner à la sphère motrice du comportement.

\section{Relation attitude et comportement: prédictivité et multifactorialité}

L'exercice sous-tend également un lien fort entre attitude et comportement «quel comportement cela aurait-il tendance à provoquer chez vous? » adoptant ainsi le paradigme théorique qui fait valoir la prédictivité des comportements par les attitudes tout en méconnaissant la multifactorialité de l'attitude. Ainsi, le postulat sous-jacent semble être une relation linéaire entre une seule attitude et le comportement qui lui serait associé, comme le montre l'usage du singulier dans les questions (quelle attitude et quel comportement?).

\section{Des pratiques contrastées chez deux enseignantes expérimentées à partir du même manuel}

Nous observons deux enseignantes d'économiegestion, expérimentées pour l'enseignement mais novices pour les savoirs issus de la psychologie, enseignant la relation entre attitude et comportement et la notion d'attitude.

Les deux enseignantes font usage du premier exercice du manuel: Différence entre attitude et comportement. Il s'agit de trois images où l'on voit une sculpture en aluminium, une femme avec les lèvres percées par une flèche, et une photo après le passage d'un tsunami. Trois questions sont associées à ces photographies a) De quoi s'agit-il? b) Que vous dites-vous ou quel jugement portez-vous spontanément face à ces photos? c) Quel comportement cela aurait-il tendance à provoquer chez vous? Selon la logique de la démarche OAC ces trois questions correspondent à la phase d'observation.

Elles ajoutent toutes les deux des documents complémentaires au manuel. 
Nous considérons les modes de construction de ces savoirs dans la classe soit la topogenèse (place respective de l'enseignante et des élèves) et la manière dont les enseignantes se confrontent aux obstacles épistémologiques liés à la nature pluriparadigmatique des savoirs en psychologie.

\section{Madame A}

L'enseignante, après avoir commenté un schéma qui reprend les différentes notions abordées qui peuvent influencer le comportement (facteurs socioculturels et psychologiques, comportement etc.), invite les élèves à utiliser le manuel (Guittard et al., 2005a) pour le premier exercice visant à distinguer attitude et comportement et comportant des questions qui permettent de construire le lien de prédictivité entre attitude et comportement. Elle explore ensuite ce lien au moyen d'un cas issu du guide pédagogique dans lequel une personne inconnue se présente (« on me présente quelqu'un »). Elle fait enfin usage d'un document, issu du $\mathrm{CRCOM}^{1}$, mettant en scène une situation d'embauche pour travailler sur les trois composantes de l'attitude (cognitive, affective et conative ou comportementale).

\section{Pseudo-dévolution et ostension directe}

Madame A. s'efforce de dévoluer (Brousseau, 1986) en insistant sur l'activité personnelle des élèves mais elle leur laisse peu de temps pour faire leurs observations à partir de l'exercice du manuel et envisage de prendre en charge la réponse aux questions:

P91 mettez vos réponses en commun avec celles de vos camarades non ce que je vais faire on va le faire à l'oral ce sera beaucoup plus vivant bon vous allez réfléchir à ces trois premières questions et ensuite je vous interrogerai à l'oral d'accord et je vous donnerai ma proposition

À plusieurs reprises (P91, P150, P152), elle semble hésiter entre deux méthodes: la méthode OAC préconisée par le programme qui demande de partir des observations des élèves, de leurs analyses pour conceptualiser et la méthode expositive où l'enseignant présente un savoir déjà construit à des élèves qui le reçoivent. Dans cet extrait, l'ostension directe, correction d'un exercice sans y intégrer les réponses des élèves, coexiste avec une demande de construction de définition par les élèves de la notion d'attitude:

P150 est-ce que vous souhaitez alors là je ne sais pas comment procéder mais est-ce que vous souhaitez vous faire un récapitulatif tel que je l'ai fait les différentes attitudes que l'on peut trouver en forme de tableau [...] soit vous faites un tableau qui correspond à vos réponses

\section{E151 la conclusion}

E152 oui [...] en conclusion ça me paraît un peu bizarre d'écrire conclusion quand on n'a rien écrit avant, on peut dire vous marquez que les attitudes varient selon qui voilà vous le notez selon les individus deux points nous manifestons des attitudes à propos de presque tout est-ce que vous êtes d'accord avec ceci des personnes de ce que nous voyons.... je finis la phrase que ce soit consciemment ou pas donc à partir de ce constat on vous demande donc de donner une définition du mot attitude je vous écoute qu'est-ce qu'on pourrait dire essayez de trouver une définition du mot attitude que vous noterez dans le répertoire

L'enseignante ajoute une étude de cas issue du guide pédagogique « on me présente quelqu'un » qui prévoit de distinguer des exemples d'attitude comme "je suis méfiante à l'égard de cette personne » et de comportement en découlant comme « je ne lui parle pas » ou « je l'agresse verbalement $»$. Certaines interactions semblent permettre la co-construction avec les élèves de la relation entre attitude et comportement:

E221 l'attitude c'est un état d'esprit

P222 dans lequel je me mets peut-être oui et le comportement ça va être

E223 la réaction

P224 la réaction vous y êtes

Dans d'autres échanges, la différence entre les notions devient confuse et le lien peu explicite. Le terme " réaction » peut caractériser à la fois attitude (E191, P192) et comportement (E189) et la différence devient essentiellement temporelle, l'un est immédiat (P192), l'autre général (E193):

P188 l'attitude ça pourrait être un état d'esprit peutêtre je ne sais pas

E189 et le comportement c'est la réaction 
P190 la réaction oui Samuel c'est pas mal ce que tu avais dit est-ce que vous sentez qu'il y a une différence entre attitude et comportement

E191 c'est très court l'attitude

P192 c'est juste de la réaction immédiate et le comportement serait une réaction

E193 plus générale

Cet enchevêtrement déstabilise Madame A et la conduit à procéder par ostension directe en dictant une définition d'attitude empruntée au programme et également présente dans le guide pédagogique sans lien avec les échanges précédents:

P194 donc vous vous allez marquer l'attitude c'est une prédisposition mentale pour porter un jugement vous êtes d'accord avec ceci [...] l'attitude est généralement stable et répétitive dans le temps pour résumer je pourrais dire que l'attitude est un état d'esprit et l'état d'esprit va se traduire grâce à un comportement

La dévolution aux élèves de la différence entre attitude et comportement semble impossible alors même que la maîtrise des savoirs est fragile chez l'enseignante. Parfois, elle annonce la distinction comme évidente, parfois elle bute sur l'écueil de la synonymie usuelle:

P170 c'est pas difficile pour le définir [...] donnezmoi un exemple donnez-moi une situation

[...]

P174 alors je comprends pas pourquoi les éditeurs ils ont fait le distinguo c'est ridicule

Certains essais de dévolution n'aboutissent qu'à un jeu de questions-réponses dans lequel les élèves sont sollicités et mettent en lien la variabilité des attitudes selon les personnes avec la variabilité des perceptions étudiées lors d'un cours précédent:

P144 [...] alors on a fait l'analyse des trois photos on vous dit efforcez-vous d'en tirer des conclusions [...] faites moi des conclusions oui

E145 selon les personnes le comportement....

P146 oui plus fort mais c'est

E147 par rapport aux personnes comment on dit par rapport les attitudes elles changent selon les personnes

P148 en fonction de l'individu [...] nous aurons des attitudes différentes à votre avis pourquoi donnez-moi des

E149 on n'a pas les mêmes perceptions
P150 voilà je vais essayer de faire le lien vous n'avez pas tous les mêmes perceptions vous n'avez pas tous les mêmes sensations vous y êtes les œuvres d'art il y a tellement d'œuvres d'art ça peut plaire à certains et déplaire à d'autres oui

L'enseignante institutionnalise la relativité de l'attitude à partir de l'exercice du manuel et de l'analyse d'un élève. Si le programme ne prévoit pas la construction de ce savoir, l'enseignante accueille la réponse de l'élève et active la mémoire de la classe. Elle la réfère à la relativité de la perception, objet du programme précédemment construit et au service de la compréhension des différents facteurs susceptibles d'influencer les situations de communication.

Les essais de dévolution de Madame A se soldent par une mise en activité des élèves qui ne leur permet qu'en partie de s'engager dans la responsabilité d'acquérir le savoir visé (Brousseau, 1998) tant la confusion existe entre savoirs exacts et inexacts. L'offre de dévolution du manuel est transformée par Madame A. en pseudo-dévolution que nous entendons comme une juxtaposition de mise en activité des élèves et de cours expositif. Ayant des difficultés dans la maîtrise des notions, elle échoue à les faire construire par ses élèves. Elle procède par ostension directe sans lien avec les échanges langagiers et institutionnalise des savoirs enseignés à la fois concordants et discordants avec les savoirs de référence de la psychologie sociale.

\section{Prévalence du sens commun, relation linéaire attitude-comportement et prise en compte des comportements non verbaux}

\section{Relation attitude et comportement: prédictivité et multifactorialité}

L'enseignante A passe d'une relation de prédictivité entre attitude et comportement (P120) à une confusion entre attitude et comportement, comme dans le sens commun :

P120 donc ça influence certainement un certain comportement une attitude

P126 de la compassion oui vous avez compris pour chacune de ces photos vous avez porté un jugement et quelle attitude avez-vous adopté si je reprends la photo

À qui c'est qui m'a dit que c'était laid heu voilà donc quelle attitude vas-tu adopter à ton avis 
E127 pas regarder

$\mathrm{P} 128$ voilà à la limite tu vas passer enfin tu ne vas pas la regarder

Entre E126 et E128 le jugement (synonyme dans cet exercice d'attitude) est la compassion et mais « l'attitude » adoptée, que l'élève propose en E127, est en fait un comportement moteur « ne pas regarder $»$.

L'étude de cas issue du guide pédagogique ( me présente quelqu'un ») consiste à donner un exemple d'attitude et de comportement dans une situation quotidienne de communication (cf. également plus haut, échanges E 221, E224):

P210 oui donc donnez-moi un exemple sur ce que vous pensez de cette personne qu'est-ce que ça peut être

E213 il est bien habillé

P214 bien habillé d'accord quel va être votre comportement s'il est bien habillé

E215 je vais être plus réceptif à ce qu'il va me dire

Cette fois, la relation de prédictivité entre attitude et comportement est nettement établie (E213, E 215). De plus, le comportement envisagé n'est plus seulement un comportement moteur mais un comportement non verbal (E219, P220):

P218 supposons qu'ici attitude vous avez un sentiment [...] vous voyez quelqu'un qui ne vous plaît pas votre comportement c'est lequel vous allez pas vers lui soyez simple dans la cour de récréation s'il ne vous plaît pas qu'est-ce que vous allez... quel va être votre comportement E219 on l'ignore

P220 vous l'ignorez vous ne lui parlez pas

Cependant, la relation entre les deux notions apparaît essentiellement comme linéaire. À une attitude (quelqu'un qui ne nous plait pas) correspond un comportement (on ignore cette personne).

Définition attitude: synonymie et sens commun, polysémie et pluriparadigmatisme

Pour construire les composantes de l'attitude, Madame A introduit le récit d'un entretien d'embauche. L'exercice prévoit de retrouver à travers le récit les trois composantes de l'attitude (affective, cognitive et conative) de Jean, consultant des ressources humaines. Madame A. fait un amalgame entre la dimension conative (ou comportementale) de l'attitude soit la prédisposition à agir (P304) et le comportement observable (P308):

P304 attention c'est les prédispositions de qui de Jean qu'est-ce qu'il va faire quelle va être son attitude

P306 mais si Jean connaît le profil type du candidat celui-ci a priori correspond le candidat au profil type oui Jean à une impression favorable il est content de ce candidat comment va-t-il agir

E307 il dit non

P308 il dit non oui donc prédispositions à agir je traduis qu'est-ce qui se passe

E309 il ne retient pas la candidature

P310 Jean ne retient pas ce candidat

La réaction finale est un comportement (P306) et non une des composantes de l'attitude. Cette ambiguité est rendue possible par la reformulation du guide pédagogique (Guittard et al., 2005b) qui semble réduire l'attitude à sa composante conative (ou comportementale) (cf. p. 194 « l'attitude c'est une prédisposition mentale pour porter un jugement $»$ ).

Dans la phase interactive, Madame A mêle les énoncés exacts et les confusions dans la mesure où elle est en difficulté pour discriminer à partir des propositions des élèves ce qui est de l'ordre de l'attitude ou du comportement.

\section{Madame B}

Le cours de Madame $B$ porte sur la définition d'une attitude. Elle initie son cours par une séance de travaux dirigés (non observée) où elle demande aux élèves de traiter le premier exercice du manuel: différence entre attitude et comportement. Le cours observé débute avec la correction de cette activité. Elle ajoute un texte scientifique (issu du CRCOM).

\section{Ostension directe, déguisée et maïeutique}

Madame B. s'efforce de procéder par dévolution; elle s'appuie sur les réponses des élèves pour faire avancer la construction du savoir visé: 
P2 par rapport à ce qui vient d'être dit vous pouvez commencer à remplir le tableau moi j'ai marqué quelque chose mais je préfère que l'on parte de ce que vous avez vu

Elle corrige l'exercice en interrogeant un à un les élèves de la classe avec un rythme de questionnement rapide. Si elle montre aux élèves les définitions des notions par ostension directe, elle s'appuie sur la concertation avec eux et ses propres observations des réponses des élèves au cours du TD:

P4 première photo on vous demandait de voir qu'est ce que c'est

E5 un animal

P6 représentation d'un animal mais avant de dire cela qu'est ce que c'est

E7 une sculpture moderne

Sous forme de maieutique, elle valide une à une les réponses des élèves:

P8 d'accord alors effectivement on va dire une sculpture

P9 on vous demandait ensuite quel est votre jugement

E10 c'est pas esthétique

E12 original

E14 ça me laisse indifférente

P15 déjà on a des jugements complètement différents

[...] donc ça c'est du jugement que vous pouvez porter à partir de là est-ce que vous avez réussi à établir des comportements

Elle cherche à ce que l'action de l'élève ne soit produite que par les nécessités du milieu et par ses connaissances, et non par l'interprétation des procédés didactiques du professeur. Cependant les obstacles véhiculés par les savoirs, l'obligent le plus souvent à procéder par maïeutique pour maintenir l'objectif de sa séance.

Dans cette élaboration commune, l'enseignante occupe une place importante. Même si les élèves sont invités à construire la notion, ils suivent un chemin préalablement tracé.

Madame B adjoint un texte scientifique (issu du CRCOM) dans le domaine du management qui vient compléter l'exercice du manuel: "Lorsqu'on connaît l'attitude d'un individu concernant un objet donné: une voiture, un patron, les syndicats ou l'informatique, on peut prédire comment il va probablement se comporter face à cet objet mais aussi par extension face à de multiples situations impliquant divers aspects de son environnement. Le concept d'attitude est une construction hypothétique et non une donnée brute observable ». Source: " Management, aspects humains et organisationnels, Aubert et al. $2002 »$.

La construction du savoir s'organise donc à partir des échanges sur l'exercice du manuel et du texte d'Aubert et al. dans lequel le savoir est en partie visible avec la notion de "donnée brute observable » et en partie caché au regard des élèves et reste à élaborer:

P97 (...) à partir de là on essaiera de définir ce que c'est qu'une attitude

Nous parlons d'ostension déguisée (Berthelot \& Salin, 1992) dans la mesure où cette proposition d'enseignement place professeur et élèves dans une fiction: celle que l'élève construit le savoir alors que, dans l'extrait donné aux élèves, il lui est à la fois montré et masqué.

Même si elle recourt à de l'ostension déguisée, maîtrisant le savoir, elle peut en partager la construction avec ses élèves. Les savoirs qu'elle institutionnalise sont concordants avec ceux de la psychologie sociale.

P144 on fonction de tout ce que l'on vient de faire pouvez vous essayer de donner une définition de l'attitude

E145 façon de se comporter

P146 la façon de se comporter est ce que ça c'est

l'attitude

E147 non c'est le comportement

E150 ensemble de jugement qui va porter sur des objets qui s'exprime plus ou moins ouvertement

P151 qu'est ce qu'on va préciser à quelqu'un qui ne connait pas la définition donc déjà si je lui dis sur des objets qu'est ce qu'il va penser

E152 la table

P153 on peut préciser entre parenthèses des fameux objets

E154 personnes situation choses

Ensuite, Madame B. institutionnalise des savoirs appropriés et procède par reconstitution de la co-construction nourrie des échanges et des prises de notes des élèves: 
P 155 donc on peut compléter cette définition mais on commence par partir de ce que vous avez écrit

E157 l'attitude c'est quelque chose d'abstrait que l'on peut porter sur des objets et qu'on matérialisera par un comportement.

P 158 oui j'aurais rajouté que l'attitude est un ensemble de jugements de tendances un état d'esprit envers un objet mais c'est très bien il faudra revenir aussi sur le mot abstrait.

Assimilation attitude et jugement, relation linéaire attitude-comportement et définition adéquate d'attitude

\section{Relation attitude et comportement: prédictivité et multifactorialité}

Dans la logique du manuel, l'enseignante confond attitude et jugement:

P15 déjà on a des jugements complètement différents [...] donc ça c'est du jugement que vous pouvez porter à partir de là est-ce que vous avez réussi à établir des comportements

P97 quelle est la différence entre un jugement et un comportement

En revanche, une élève propose comme exemple de jugement «E14: ça me laisse indifférente » puis comme exemple de comportement «E18: je vais rester indifférente ». Elle rapproche ainsi jugement et comportement en congruence avec le programme qui indique que « l'attitude est une prédisposition à porter un jugement ou à adopter un comportement » et avec les définitions d'Allport (1935) ou d'Oskamp (1977). Pourtant, l'enseignante persiste à demander à l'élève un exemple de comportement moteur, qu'elle obtient:

P19 rester indifférente tu l'as mis dans comportement il me semble que tu as mis quelque chose derrière

E20 dans un musée je vais continuer

$\mathrm{Au}$ cours de la correction collective de l'exercice, l'enseignante s'appuie sur le tableau proposé par le guide pédagogique (Guittard et al., 2005b). Elle distribue un polycopié à ces élèves pour noter la correction, dans un tableau (tableau 1, extrait du guide pédagogique):

\begin{tabular}{|c|c|c|}
\hline Les objets & $\begin{array}{c}\text { Votre jugement, ce que } \\
\text { vous en dites }\end{array}$ & $\begin{array}{c}\text { Les comportements } \\
\text { qui peuvent en } \\
\text { découler }\end{array}$ \\
\hline $\begin{array}{c}\text { Un objet, } \\
\text { une } \\
\text { œuvre } \\
\text { d'art }\end{array}$ & $\begin{array}{c}\text { Aime ou n'aime pas } \\
\text { indifférence } \\
\text { jugement sur l'auteur }\end{array}$ & $\begin{array}{c}\text { Envie d'acheter } \\
\text { Envie de se mettre à } \\
\text { la peinture }\end{array}$ \\
$\begin{array}{c}\text { Une } \\
\text { femme } \\
\text { avec une } \\
\text { flèche à } \\
\text { travers la } \\
\text { langue }\end{array}$ & $\begin{array}{c}\text { attitude liée à une } \\
\text { émotion }\end{array}$ & $\begin{array}{c}\text { Céta peurner faire regard } \\
\text { provoquer un juge- } \\
\text { ment négatif }\end{array}$ \\
\hline
\end{tabular}

Tableau 1 : correction de l'exercice 1 dans le guide pédagogique

Le rapport entre jugement, attitude et comportement est problématique comme le montre cet échange:

E38 Madame si on a dit indifférent on le note ou dans le jugement ou comportement

P39 d'après toi

E40 moi je le mettrais dans comportement car si je suis indifférente je passe

P4l d'accord comme on a dit tout à l'heure que c'était le fait de continuer son chemin.

L'institutionnalisation du savoir opéré par l'enseignante renforce l'idée qu'un comportement est lié à une activité motrice observable. L'indifférence est effectivement une attitude inobservable, inférable à partir de manifestations observables (continuer son chemin). Elle apparaît dans la colonne « jugement » dans la logique de la synonymie jugement/attitude introduite par le guide. Cette synonymie erronée en psychologie sociale introduit de la confusion entre les termes. Cette confusion est largement entretenue par la correction notée par les élèves dans le tableau de correction où jugement apparait à la fois dans la colonne désignant des attitudes (colonne 2, « votre jugement, ce que vous en dites ») et dans celle présentant des comportements (colonne 3, «provoquer un jugement négatif $»$ ).

En outre, les comportements proposés sont des composantes comportementales de l'attitude, par exemple envie d'acheter, le comportement étant l'achat à proprement parler. 
Dans la pratique de classe, l'enseignante et les élèves butent sur cette juxtaposition de savoirs justes (caractère observable du comportement) et d'ambiguïtés (confusion attitude/jugement).

Toutefois, Madame B parvient à faire construire la différence entre attitude et comportement en évitant l'écueil du sens commun tout en utilisant un exemple issu de la vie quotidienne proposé par un élève (E115):

P108 est ce que l'on va l'exprimer ou pas est-ce que quelqu'un extérieur va le voir

[...]

E115 par exemple par rapport à une voiture on a un comportement

P116 prenons l'exemple de la voiture qu'est ce qui va matérialiser ton attitude

E117 si un propriétaire la chouchoute sa voiture tout les matins on va le voir

[...]

E 123 la façon d'être de réagir mais on est dans le comportement

P124 on a bien compris le comportement on a vu que c'est ce qui va être observable mais qu'est ce qui entraine le comportement

[...]

E127 mais madame, il aime sa voiture [...] son comportement va être de chouchouter

P128 on peut imaginer que le comportement soit en fonction

E129 d'une attitude

P130 on la voit ou on ne la voit pas

E131 on soupçonne l'attitude en fonction du comportement

Les élèves s'écartent de leurs conceptions familières où attitude équivaut au comportement. Grâce à cet exemple, Madame B va faire construire l'idée que l'attitude ne se voit pas mais s'infère depuis un comportement (E123, E131).

Définition attitude: synonyme et sens commun, polysémie et pluriparadigmatisme

Dans sa construction de définition de la notion d'attitude, elle se détache bien du sens commun pour construire une définition conforme au savoir de psychologie. Cependant, elle construit ici un lien déterministe entre un comportement et son attitude et néglige de ce fait la multifactorialité des influences d'un comportement et de la multiplicité des attitudes qui influencent un comportement (cf. ci-dessus, E127, P128, E129).

À la fin de son cours, elle institutionnalise la définition de l'attitude, qui consiste à une reformulation de la définition du guide pédagogique: P158 « le terme attitude désigne un ensemble de jugements, de tendances, un état d'esprit envers un objet [...] L'attitude n'est pas identifiable a priori, elle exprime ce que l'individu ressent et elle peut pousser à certains comportements; l'attitude est donc une prédisposition à agir. L'attitude permet de mieux comprendre, d'anticiper et même de tenter de modifier les comportements ». Cette définition est congruente avec le savoir de psychologie sociale et les programmes, et n'est pas référée aux théories de référence.

Madame B met ses élèves en situation d'explorer le savoir à construire. Dans la phase interactive, elle accueille leurs réponses qu'elle valide ou non dans la mesure où elle est en mesure de discriminer à partir des propositions des élèves ce qui est de l'ordre de l'attitude ou du comportement. Elle accueille la parole des élèves avec un guidage très serré de l'ordre de la maïeutique.

\section{Discussion-conclusion}

Nous nous intéressons aux transformations des savoirs psychologiques dans différentes étapes de la chaîne transpositive et aux pratiques d'enseignement de deux professeures expérimentées d'économie-gestion.

Nous avons montré deux obstacles identifiés avec l'analyse a priori des savoirs quant à la construction des savoirs: définition de sens commun et polysémie dans un cadre pluriparadigmatique d'une part, et multifactorialité et relation de prédictivité entre attitude et comportement, d'autre part. Le troisième obstacle correspond à la mise en ouvre de la démarche Observation-Analyse-Conceptualisation (OAC).

Le premier obstacle porte sur la polysémie du terme d'attitude et l'assimilation problématique entre attitude et comportement existant dans le sens commun. 
Le programme propose une définition unidimensionnelle du concept d'attitude (cf. sens et portée de l'étude) et en même temps détaille les caractéristiques de ce concept en empruntant à un autre paradigme trois composantes de l'attitude (cf. notions et contenus à construire). La présentation du concept et ses caractéristiques relève d'une superposition des paradigmes qui peut créer de nouveaux obstacles en particulier une confusion entre composante comportementale (conative) de l'attitude et comportement.

Suivant la proposition du manuel, Madame B. assimile attitude et jugement, ce qui la conduit à minorer les aspects non verbaux du comportement et à circonscrire le comportement à son aspect moteur. Madame A. aborde les aspects non verbaux du comportement mais se perd dans la confusion commune entre les deux notions. Mais il existe une différence majeure entre les deux enseignantes. Tout au long de la séance, Madame A. ne parvient pas à se détacher durablement du sens commun d'attitude et n'ouvre que peu d'espace pour que ses élèves assimilant attitude et comportement s'affranchissent de leurs concepts spontanés (Vygotki, 1985). Madame B. en dépit des confusions entre attitude et jugement ouvre cet espace en mettant en valeur les spécificités de l'attitude au sens de la psychologie dans les réponses des élèves.

Le deuxième obstacle porte sur le lien entre les deux concepts d'attitude et comportement. En psychologie, il n'existe pas de prédictivité linéaire entre une attitude et son comportement associé. Plusieurs attitudes peuvent être à l'origine (ou non) d'un comportement. Si le programme et le manuel affirment la relation de prédictivité, ils la présentent sous une forme linéaire et non multifactorielle. Madame A fluctue entre relation de prédictivité et assimilation au sens commun. La confusion que Madame B opère entre attitude et jugement rend problématique la construction de la relation de prédictivité entre attitude et comportement.

Nous montrons que le concept de transposition didactique est pertinent pour des savoirs pluriparadigmatiques (Dollo, 2005) issus de la psychologie dans la mesure où elle permet au chercheur de rendre compte de leurs pérégrinations au cours des différents relais entre décisions de la noosphère et pratiques de classe.
Nous constatons surtout que les questions génératrices du savoir au sens de Chevallard, traitées dans le cadre de plusieurs paradigmes en psychologie sont absentes. Or, dans l'optique de la transposition didactique, la référence aux concepts de la psychologie permet, par la conceptualisation, de poser les questions relatives à la communication. Or, " pour un esprit scientifique, toute connaissance est une réponse à une question. S'il n'y a pas eu de question, il ne peut y avoir connaissance scientifique. Rien ne va de soi. Rien n'est donné. Tout est construit » (Bachelard, 1938, p. 14). Ces questions auxquelles correspond le savoir enseigné sont toujours débattues dans la communauté des psychologues. Cependant, les cours observés permettent difficilement aux élèves de se les poser et de tenter d'y apporter collectivement des réponses: «Pourquoi opérer une distinction entre attitude et comportement ?»; «Y a-t-il une relation de prédictivité entre attitude et comportement?» restent des questions non prises en charge au sein de la classe.

L'exercice de transposition est particulièrement difficile pour ces savoirs de psychologie transportés dans le second degré, contexte éloigné de leur lieu de production habituel, avec pour injonction de les mettre au service de l'analyse et de la compréhension de situations de communication, sans se référer aux théories qui les fondent, alors que la question de leur transposition au besoin de l'enseignement dans des filières technologiques de la gestion n'est pas très approfondie dans les programmes.

Le programme préconise sans exclusive la méthode OAC sans toutefois préciser ce que le terme conceptualisation recouvre. Cette imprécision renvoie aux autres relais de la chaîne transpositive la responsabilité de l'interpréter. Les lettres dans le manuel sont explicitement référées à la méthode sans qu'on puisse toutefois distinguer la différence opérée entre l'analyse et la conceptualisation dans la formulation des questions.

Les enseignantes sont donc confrontées à une reproduction des habitudes de la filière en assimilant conceptualisation et synthèse, partie ultime de la démarche inductive. Cette démarche inductive était compatible avec les compétences professionnelles travaillées en STT (Sciences et Technologies Tertiaires), alors que les nouveaux savoirs pluri- 
paradigmatiques et théoriquement fondés y sont peu propices. En effet, on «ne peut répondre à ces questions par une démarche inductive reposant sur l'observation de son environnement immédiat * (Beitone, 2007, p 67) dans la mesure où « l'observation première est toujours un premier obstacle pour la culture scientifique. En effet, toute observation première [...] est pittoresque, concrète, naturelle, facile. Il n'y a qu'à la décrire et à s'émerveiller. On croit alors la comprendre " (Bachelard, 1983, p. 19). Nous suivons Dollo qui affirme pour les Sciences Économiques et Sociales que «le caractère pluriparadigmatique des sciences de référence est l'un des éléments essentiels qui doit conduire à interdire de partir des faits et de l'observation, mais bien d'objets de savoirs construits et repérés comme produits par une communauté savante $»$ (Dollo, 2005, p. 8).

Certes, les activités proposées par le manuel et les exercices ajoutés par les enseignantes permettent de faire valoir l'intérêt de recourir aux concepts d'attitude pour expliquer des comportements de communication. Mais ces concepts en psychologie sont trop denses pour s'accommoder d'une simplification qui rend la relation linéaire ou de définitions de sens commun. Les concepts spontanés (Vygotski, 1985) qui confondent dans un même sens attitude et comportement sont trop stabilisés pour permettre une réorganisation cognitive en faveur d'une appropriation des concepts scientifiques, qui demeure extérieure au système cognitif des élèves.

Par conséquent, un compromis est trouvé par les enseignantes qui mettent en œuvre des techniques didactiques d'ostension directe et "déguisée » (Berthelot et Salin, 1992) et la maieutique (Parlebas, 1980). Le manuel assigne à l'enseignant le rôle de faire réaliser les activités proposées, lui épargnant la charge d'une transposition didactique. Ainsi, les enseignantes voient leur rôle restreint à souffler le savoir, étant donné que l'adidacticité des activités proposées est insuffisante et rend difficile l'existence du collectif classe sous sa direction au service de la construction du savoir - ce qui se différencie d'une simple mise en activité des élèves. L'ostension directe, déguisée et la maieutique semblent en conséquence les solutions que ces enseignantes d'économie-gestion mobilisent en réponse aux difficultés de la tâche professionnelle. Toutefois, l'ostension des concepts par les enseignantes et le questionnement de type maïeutique peuvent difficilement conduire à une intériorisation des concepts par les élèves qui serait véritablement l'activité de conceptualisation.

Le concept de topogenèse, introduit en didactique des mathématiques, nous parait opérant en didactique de l'économie-gestion, tant son analyse a priori dans les manuels scolaires que l'analyse des processus topogénétiques en classe. Il permet en effet de rendre compte de mécanismes d'adoption ou de résistance aux principes d'une réforme en acte. Nous montrons ainsi que la topogenèse est un outil conceptuel qui permet de comprendre l'activité de l'enseignant et des élèves dans le cadre d'un enseignement pluriparadigmatique mettant en jeu des savoirs issus des sciences humaines, en particulier la psychologie. 


\section{NOTES}

1. Réseau national de ressources pédagogiques en Communication-Organisation-Management

\section{RÉFÉRENCES}

Allport, G. (1935). Attitudes. In C.M. Murchison (Ed.), A Handbook of Social Psychology (p. 798-844). Worchester, MA: Clark University Press.

Bachelard, G. (1983), La formation de l'esprit scientifique, Paris: Librairie Philosophique J. Vrin. (original publié 1938).

Barth, B-M. (1993). La détermination et l'apprentissage des concepts. In J. Houssaye (Ed.), La pédagogie: une encyclopédie pour aujourd'hui (p. 275-288). Paris: ESF.

Beitone, A. (2007). Pour en finir avec la pédagogie inductive. Cahiers pédagogiques, 449, p. 66-68.

Berthelot, R. \& Salin, M-H. (1992). L'enseignement de l'espace et de la géométrie dans la scolarité obligatoire. Thèse de doctorat, Université de Bordeaux 1, Bordeaux.

Brossais, E., Panissal, N., Matheron, Y., Ludwig-Legardez, A., Legardez, A., \& Terrisse, A. (2007). Évolution de l'enseignement d'information et communication au lycée technologique français. Étude des manuels scolaires de Sciences et Technologies de la Gestion. In M. Lebrun (Ed.), Le manuel d'ici et d'ailleurs, d'hier à demain. Québec: Presse de l'Université du Québec (livre et CD-Rom).

Brousseau, G. (1986). Fondements et méthodes de la didactique des mathématiques. Recherches en didactique des mathématiques, 7.2, 33-115.

Bruillard, E. (2005), Manuels scolaires, regards croisés. Caen: SCEREN-CRDP.

Chevallard, Y. (1985), La transposition didactique: du savoir savant au savoir enseigné. Grenoble: La Pensée Sauvage.

Dollo, C. (2005) Généricité/spécificité d'un concept: La transposition didactique en sciences économiques et sociales. Revue suisse des sciences de l'éducation, 1, p. 85-102.

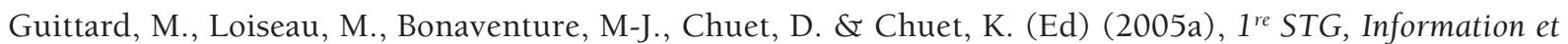
Communication. Paris: Bertrand- Lacoste.

Guittard, M., Loiseau, M., Bonaventure, M-J., Chuet, D. \& Chuet, K. (Ed) (2005b). $1^{\text {re }}$ STG, Information et Communication. Guide pédagogique. Paris: Bertrand- Lacoste.

Legardez, A. (Coord.) (2002). Circulation et reproblématisation des savoirs dans les enseignements économiques, sociologiques et de gestion. (Rapport de recherche) Aix-Marseille: INRP et IUFM.

Legardez, A. (2004). Transposition didactique et rapports aux savoirs; l'exemple de questions économiques et sociales, socialement vives. Revue Française de Pédagogie, 149, p. 19-28. 
Legardez, A. (2005). Quels critères d'évaluations pour les enseignements d'économie? Une approche sociodidactique des enseignements économiques des lycées. Questions Vives, 6, p. 145-150.

Michelik, F. (2008). La relation attitude-comportement: un état des lieux. Ethique et économique. 6 (1) Consulté le 3 septembre $2010 \mathrm{http} / / /$ hdl.handle.net/1866/3417

Osgood, C. E., Suci, G. J., \& Tannenbaum, P. H. (1957), The measurement of meaning, Urbana, IL: University of Illinois Press.

Oskamp, S. (1977), Attitudes and opinions. Englewood Cliffs, N.J.: Prentice-Hall, Inc.

Panissal, N., Brossais, E. Legardez. A. \& Ludwig-Legardez. A. (2007). Les manuels dans la réforme STG: nouvelles normes, évolutions et résistances. Communication présentée à la $3^{e}$ journée Pierre Guibbert « Les manuels scolaires entre normes et controverses: continuités, aléas, avatars», IUFM Montpellier, France.

Park, R.E., Burgess, E. (1921), Introduction to the Science of Sociology. Chicago : University of Chicago Press.

Parlebas, P. (1980). Un modèle d'entretien hyperdirectif: la maieutique de Socrate. Revue Française de Pédagogie, 51, p. 4-19.

Programme de l'enseignement d'information et communication en classe de première de la série Sciences et technologies de la gestion (2004). Consulté le 30 juin 2010 http://www.education.gouv.fr/bo/2004/hs1/ MENE0400023A.htm

Rosenberg, M.J. \& Hovland, C.I. (1960). Cognitive, affective and behavioral components of attitudes. In C.I. Hovland \& M.J.Rosenberg (Eds.), Attitude organization and change (p. 1-14), New Haven, CT: Yale University Press.

Sensevy, G. (2001). Théorie de l'action et action du professeur. In J-M Baudoin et J. Friedrich (Eds). Théories de l'action et éducation. (p. 203-224). Bruxelles: De Boeck Université

Sensevy, G. (2007). Des catégories pour décrire et comprendre l'action didactique. In G. Sensevy \& A. Mercier (Eds.), Agir ensemble: L'action didactique conjointe du professeur et des élèves (p. 13-34). Rennes: Presses Universitaires de Rennes (PUR).

Stoetzel, J. (1963), La psychologie sociale. Paris: Flammarion.

Tavignot, P. (1995). À propos de la transposition didactique en didactique des mathématiques. Spirale - Revue de Recherches en Éducation, 15, 31-60.

Thomas, W.I., Znaniecki, F. (1919). The Polish Peasant in Europe and America. Volume 3: Life record of an immigrant. Boston, Mass. : Badger.

Verdelhan-Bourgade, M., Bakhouche, B. Boutan, P., \& Étienne, R. (2007). Les manuels scolaires, miroirs de la nation? Paris: L'Harmattan.

Wiggins, E. C., \& Breckler, S. J. (1992). Management of complex civil litigation. In D. K. Kagehiro \& W. S. Laupher (Eds.), Handbook of Psychology and Law (p. 77-94). New York: Springer-Verlag. 